\title{
A distributed coordination mechanism for supply networks with asymmetric information
}

\begin{abstract}
The paper analyses the problem of coordination in supply networks of multiple retailers and a single supplier, where partners have asymmetric, private information of demand and costs. After stating generic requirements like distributedness, truthfulness, efficiency and budget balance, we use the apparatus of mechanism design to devise a coordination mechanism that guarantees the above properties in the network. The resulting protocol is a novel realisation of the widely used Vendor Managed Inventory (VMI) where the responsibility of planning is at the supplier. We prove that together with the required generic properties a fair sharing of risks and benefits cannot be guaranteed. We illustrate the general mechanism with a detailed discussion of a specialised version, assuming that inventory planning is done according to the newsvendor model, and explore the operation of this protocol through computational experiments.
\end{abstract}

Keywords: Supply chain management, Vendor Managed Inventory, game theory, mechanism design, asymmetric information

\section{Introduction}

Supply networks are large and complex systems, characterised by the existence of numerous competitive enterprises, dynamic structures, uncertain knowledge, asymmetric information structure, and difficult planning and decision making problems. The uncoordinated actions in such a system lead to suboptimal performance, exemplified in a simple case by the well-known prisoners' dilemma. In supply networks this phenomenon is called double marginalisation: since every enterprise concerns its own profit when making decisions, the aggregate benefit is in general lower than if the enterprises were vertically integrated and centrally controlled. This suboptimality manifests itself in waste of materials, labour, energy and other environmental resources, and eventually causes significant financial losses, too. Hence, analysing the interactions of autonomous enterprises and designing coordination methods that are applicable and useful also in the industrial practice are some of the most compelling challenges of operations management today (Váncza et al., 2011).

In a vertically integrated supply network with multiple retailers and a supplier, centralising the replenishment and inventory management decisions at 
the supplier's side is advantageous compared to the situation where each retailer has to decide individually. This centralisation approach is called risk pooling, and it is proved to result both in lower average inventory levels and safety stocks (Simchi-Levi et al., 2000).

In order to use the idea of risk pooling in vertically non-integrated networks, the Vendor Managed Inventory (VMI) business model is applied frequently in the practice. In this case, the supplier takes all risks and full responsibility for managing a one-point inventory, while it tries to fulfil the demand occurring at the retailers (Simchi-Levi et al., 2000). In this situation, it is hard to decide what is the reason if a network performs poorly: were the forecasts unreliable, or was the planning inappropriate? If the retailers are not faced with the consequences of an imprecise forecast directly, they are not inspired to increase their efforts in accurate forecasting. On the contrary, they even have incentives to distort the forecasts, and tend to overplan demand and forward too optimistic values towards the supplier, in order to avoid lost sales. Alternatively, if the retailers are rewarded for overperforming the plans, then they tend to underestimate the demand, and hope that the supplier can still fulfil a higher realisation. In both cases, the selfish distortion of information introduces additional uncertainty into the demand forecasts, and leads to higher operational costs or higher lost sales.

We have observed both of the above phenomena when working on improving the performance of real supply chains. In a manufacturing domain, the supply network under study produces mass products like lighting sources and appliances. The products are marketed and sold on local markets by distribution centers throughout Europe. The distribution centers are autonomous business units with their own objectives, business plans and special knowledge of the local markets, hence they prepare the demand forecasts. On the other hand, so as to exploit economies of scale, production is concentrated at a focal manufacturer who is responsible for cost efficient production and making inventory replenishment decisions on behalf of the distribution centers. Operations of the factory are highly complex (for a description of its production scheduling problem, see (Drótos et al., 2009)), the actual costs of serving the distribution centers depends on a number of factors among which the quality of received forecasts is of primary impact. Our other motivating example comes from the practice of a retail trade service, where local stores are linked to a central warehouse. The distributed stores are autonomous profit centers responsible for serving their local customers. They generate forecasts for demand of various fast moving consumer and durable goods. In contrast, the responsibility for organizing supply of these goods is at the central warehouse who is connected to the suppliers and makes appropriate decisions of purchasing, replenishment and logistics. Similar to the industrial case above, processes and data of central planning are not known to the distributed retailers. The quality and reliability of their local forecasts, however, have a major impact on the efficiency of the overall system.

The literature of supply chain coordination with asymmetric information usually assumes that either the retailers' demand forecast or the supplier's cost is private information, or rarely both (for an overview, see Egri and Váncza, 
2012). In this paper, we consider that both demand forecasts and production costs are private information of rational agents, and apply the apparatus of mechanism design theory in order to analytically investigate supply coordination mechanisms that exhibit generic properties like efficiency, truthfulness or budget balance ${ }^{1}$. By exploiting the special properties of the coordination problem, we construct a truthful and efficient mechanism that can be implemented in a distributed way. The resulting VMI-type relationship specifies an appropriate information exchange and payment scheme that has a straightforward interpretation. We illustrate the general mechanism with a detailed discussion of a specialised version, assuming that inventory planning is done according to the newsvendor model, and explore the operation of this protocol through computational experiments.

The remainder of the paper is organised as follows. In Section 2, we review the related literature. Next, our general supply network coordination model is presented in Section 3. We demonstrate the approach for the particular case of the newsvendor problem in Section 4. Finally, Section 5 discusses potential applications and extensions of the method and concludes the paper.

\section{Literature review}

In this section we review the main topics that relate to our paper. Coordination with contracts aims at constructing such enforceable rules whereby the overall supply chain performance can be optimised. Game theory determines the possible results of strategic situations defined by a number of participants, their own goals and decision options. Mechanism design is a subtopic of game theory, where the aim is to influence the participants in order to achieve some preferred outcome. In fact, coordination with contracts is a special application of this approach in the field of supply chains. However, there are several achievements of mechanism design which have not been used in this context so far, thus applying them seems to be a promising research direction. Finally, information elicitation models such problems, where the participants have private information about the probability of a stochastic event. We utilise this approach in our model assuming that the demand forecasts are only known by the retailers.

Several papers discuss different contractual forms for achieving optimal supply chain efficiency, called coordination, both for VMI and non-VMI models; for overviews see (Cachon, 2003) and (Li and Wang, 2007). Yu et al. (2009) study the VMI supply between a supplier and multiple retailers assuming symmetric information. They show that the network performance can be improved with cooperative contracts, but they cannot achieve perfect channel coordination. Chen and Bell (2011) consider price-dependent stochastic demand, customer returns and a retailer-supplier pair with symmetric information. They prove that the standard buyback contract cannot coordinate the channel in this case, but a modified version with two different buyback prices can, and furthermore,

\footnotetext{
${ }^{1}$ For definitions see Section 3.
} 
it enables profit sharing between the partners. Chen and Xiao (2011) present a model for a supply chain of short life-cycle products whose prices drastically decrease in the selling season. In a retailer-supplier chain with symmetric information, they develop buyback-based contracts that coordinate the channel and provide win-win situation for the partners.

As for the asymmetric information models, Liu and Özer (2010) compare the widely used price-only, quantity flexibility and buyback supply contracts when the demand forecast information is private. They assume that the forecast is either shared truthfully or not shared at all. It is shown that in this asymmetric situation the quantity flexibility and buyback contracts are not equivalent any more, since the quantity flexibility contract may not warrant truthful information sharing and coordination. Wang et al. (2009) assume price-dependent demand and that the production cost of the supplier is private information. The paper studies several contract forms and concludes that none of them can guarantee truthful information sharing.

Recently, the game theory forums have also become interested in supply chain applications. An overview can be found in Nagarajan and Sošić (2008), which focuses on cooperative models including bargaining and coalition formation. Yu and Huang (2010) study a network with a supplier, multiple retailers and symmetric information, but assumes VMI supply. In this case, the retailers can decide about the retail prices and the advertising investments. The authors do not intend to coordinate the network, but to develop an efficient algorithm for computing the Nash equilibrium. Esmaeili et al. (2009) consider a single supplier single retailer setting with deterministic demand and symmetric information. In their model the supplier is responsible for the lot sizing decision, therefore it can be considered a VMI system. Instead of coordinating the channel, their goal is to characterise the Pareto-efficient cooperative solutions that can be used during the price negotiation between the partners. Wang et al. (2004) model a supply network with one supplier and multiple retailers, and study the setting as a non-cooperative game with symmetric information. They also consider the situation when the supplier has some strict production constraints and thus the retailers must compete for the supply. The paper also presents contracts that result in unique Nash equilibria and coordinate the network.

Mechanism design theory deals with the problem of constructing the rules of a game with incomplete (asymmetric) information in order to achieve some preferred outcome. For an overview of the classic mechanism design theory we refer to Narahari et al. (2009). One of the main achievements in this field is the Vickrey - Clarke-Groves (VCG) mechanism, which is the only one in the general model that can provide efficient and truthful behaviour, but unfortunately, it cannot guarantee the budget balance property. An other possibility is to apply the mechanism developed by d'Aspremont, Gérard-Varet and Arrow (dAGVA), which results in efficiency and budget balance. Unfortunately, the dAGVA mechanism offers a much weaker equilibrium concept than the VCG, and it also necessitates some common belief about the private information, e.g., a probability distribution (belief) about a probability distribution (forecast). We deem the availability of such fine-grained information unrealistic in a supply 
chain setting.

Feldmann and Müller (2003) model a general multi-echelon supply chain as a mechanism design problem. They apply the VCG mechanism for inspiring the partners to disclose their private information truthfully with a central, independent Supply Chain Management. They also develop a modified version of the mechanism that assumes that the private information can be later verified and the untruthful players can be penalised. Garg et al. (2005) investigates a supply chain optimization model based on the six sigma methodology. In a decentralised environment with asymmetric information, they assume a central decision maker that applies a VCG mechanism to optimize the chain and motivate the players to provide the private information truthfully. Gan et al. (2010) present a model with similar assumptions to ours. They consider a drop-shipping supply chain, where the supplier is responsible for the inventory management, but only the retailer knows the precise demand forecast. The realised demand (including the lost sales) is assumed to be common knowledge, and the authors apply mechanism design to coordinate the channel. However, there are several differences between the model of Gan et al. (2010) and our one: (i) we consider multiple retailers, (ii) our solution does not restrict flexibility with purchase commitments, (iii) we assume that the production cost is private information of the supplier, (iv) our model is more general allowing not only the newsvendor, but any other lot sizing or production planning problem, (v) we allow any kind of demand forecast, not only high or low random, and (vi) we do not assume that the supplier has any prior belief about the retailers' private information.

In our previous work (Egri and Váncza, 2011), we studied a supply network with one supplier and several retailers applying VMI supply with no lost sales allowed. We developed a coordination mechanism that gives incentives to the retailers to share the best available forecasts with the supplier, who in turn determines the production quantity and takes all consequences of any inappropriate decision. The difference between the problem examined in that paper and our present model is that here the output of the mechanism has a direct effect on the income of the retailers due to the allowed lost sales. For this reason, it is not enough to punish the retailer for an inappropriate forecast, since, intuitively, it may be worth overestimating the demand and pay penalty, if it is compensated by the increased income caused by decreased lost sales. In this case a more sophisticated incentive mechanism is required.

The information elicitation problem consists of an agent with private information about the distribution of some stochastic future event, and an other agent that is interested in this information. It can be considered "a subproblem of mechanism design, where the mechanism has no outcome to determine" (Zohar and Rosenschein, 2008). Such information elicitation problems can be handled by applying so-called strictly proper scoring rules, see e.g., Savage (1971). We briefly define this instrument here.

Let us assume a set $D$ of possible events, and $\mathcal{P}$, a class of probability measures over them. A scoring rule $S: \mathcal{P} \times D \rightarrow \mathbb{R}$ is called strictly proper, if whenever an event $\xi$ is drawn from the distribution $\theta$, then for any other $\hat{\theta} \neq \theta: \mathbb{E}_{\theta}[S(\theta, \xi)]<\mathbb{E}_{\theta}[S(\hat{\theta}, \xi)]$. With other words, the score can be minimised 
(in expectation), if it is parametrised with the real distribution of the stochastic event. ${ }^{2}$ For instance, in what follows $\theta$ will represent the demand forecast known by, while $\hat{\theta}$ the demand forecast communicated by the retailer (which are not necessarily the same).

Well-studied examples for strictly proper scoring rules are the quadratic, the spherical and the logarithmic rules (see e.g., Zohar and Rosenschein, 2008). Applying such rules to the information elicitation problem is straightforward: if the agent with the private information is penalized proportionally to a proper score, it becomes interested in creating and providing as good forecast as possible. Note that we implicitly assume that the forecast can be created free of charge; when forecasting or improving the forecast involves some cost, truthful mechanisms may not exist in general (Zohar and Rosenschein, 2008).

\section{Coordinating the supply network}

\subsection{Requirements}

We give first the generic requirements towards mechanisms aimed at coordinating a supply network that consists of autonomous agents. Note that all the requirements comply with the conditions and expectations of industrial practice. Hence, we seek mechanisms that satisfy the following desirable criteria:

Distributed implementation. The mechanism should omit any independent decision maker, thus every decision should be made by the agents. This implies the requirement for the budget balance property, i.e., all payments should be distributed among the agents.

Strong solution concept. We require that the game theoretic solution concept should be as strong as possible by avoiding any knowledge or belief of the agents about each other's private information. Our mechanism should guarantee that truthful revelation of the private information is dominant strategy for the retailers, and the optimal decision of the supplier is also dominant.

Efficiency. The mechanism should achieve a solution that is optimal according to a network-wide utility function.

Participation. In order to assure participation of the agents, the mechanism should guarantee non-negative utility or some additional profit compared to a baseline solution.

\subsection{Supply network model}

In this section we discuss a supply network model of $n$ retailer agents and a supplier agent. The model is an extension of the one presented in Egri and

\footnotetext{
${ }^{2}$ Note that in contrast to the usual notation, for convenience, we minimize the score.
} 
Váncza (2011). By omitting the assumption that lost sales are not allowed, we generalise the model, e.g., the current variant enables the application of capacity constrained planning as well.

For the sake of simplicity, we assume that the retailers are homogeneous in the sense that they have the same possible types of forecast, although this assumption can be relaxed and the results still remain valid. Retailer $i(i=$ $1, \ldots, n)$ has some private belief (forecast) about the future market demand, which is denoted by $\theta_{i} \in \Theta$. Furthermore, we denote the vector of the forecasts as $\theta=\left(\theta_{1}, \ldots, \theta_{n}\right)$. The demand is satisfied by production done at the supplier who has, in turn, private information about the cost factors. Since the exact demands $\xi_{i} \in D$ realise at some later time, only the forecasts can be considered when creating a production plan denoted by $x \in \mathcal{K}$. The production plan specifies how the demand should be satisfied, including for example production quantities, schedule and distribution. We represent the demand vector as $\xi=\left(\xi_{1}, \ldots, \xi_{n}\right)$ and the production cost at the supplier as a function of the production plan as well as of the realized demands: $c \in C=\left\{c: \mathcal{K} \times D^{n} \rightarrow \mathbb{R}\right\}$, which is a private information of the supplier. Note that we do not assume that an a priori distribution about the private information $\left(\theta_{i}\right.$ and $\left.c\right)$ is known by the other agents, i.e., we regard a situation with strict incomplete information. After the demands realise, which are considered to be commonly observable, an income arises at each retailer $i$ from the sales: $v_{i}: \mathcal{K} \times D^{n} \rightarrow \mathbb{R}$. Note that we do not restrict the demand at the different retailers in any way; they can be drawn from non-identical distributions, they can correlate or can be independent.

This model illustrated on Fig. 1 is our general framework that does not specify how to determine the production plan and how to allocate the costs and incomes among the agents. The dashed arrows denote information flow, while the solid ones represent the monetary payments. The variables and functions inside the agents are private knowledge of the given agent and are unobservable for the others.

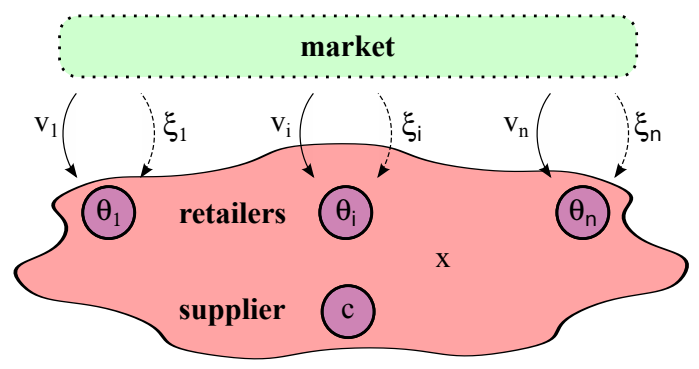

Figure 1: Supply network framework.

\subsection{Incentive mechanism}

According to the classic mechanism design theory, an independent mediator, the mechanism is required for observing the agents' actions, making the 
necessary decisions and after realisation, transferring the payments among the agents. Some recent research directions aim at omitting this mediator, which possibility we will study in the next section. For the moment, let us define the mechanism as $\mathcal{M}=\left(f, t_{1}, \ldots, t_{n}, t_{s}\right)$, where $f: \Theta^{n} \times C \rightarrow \mathcal{K}$ is the choice function determining the production plan based on the forecasts and the cost function ${ }^{3}, t_{i}: \Theta^{n} \times C \times D^{n} \rightarrow \mathbb{R}$ are the payment functions of the retailers $(i=1, \ldots, n)$, and $t_{s}: \Theta^{n} \times C \times D^{n} \rightarrow \mathbb{R}$ is the payment for the supplier.

Note two assumptions of this formulation. Firstly, this is a direct-revelation mechanism, i.e., the strategy of the agents is to share their private information (not necessarily truthfully) with the mediator. According to the revelation principle, this can be done without loss of generality (see e.g., Narahari et al., 2009). Secondly, we consider that the realised demands and the production plan are commonly observable for the agents and also for the mechanism, and the execution of the plan can be enforced at the supplier. Common knowledge of the agents is also known to the mechanism.

Now we can define the utility - the income minus the cost - for each retailer and the supplier:

$$
u_{i}\left(\theta_{i}, \hat{\theta}, \hat{c}, \xi\right)=v_{i}(f(\hat{\theta}, \hat{c}), \xi)-t_{i}(\hat{\theta}, \hat{c}, \xi)
$$

and

$$
u_{s}(c, \hat{\theta}, \hat{c}, \xi)=t_{s}(\hat{\theta}, \hat{c}, \xi)-c(f(\hat{\theta}, \hat{c}), \xi)
$$

respectively, if the agents' private information is $\theta_{i}$ and $c$, but they communicate $\hat{\theta}_{i}$ and $\hat{c}$ instead. Each agent intends to maximize its own expected utility.

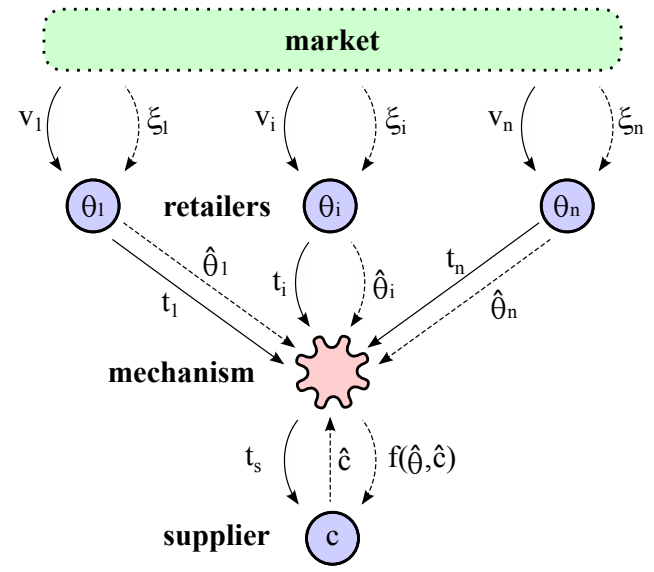

Figure 2: Supply network in a classic mechanism design setting.

\footnotetext{
${ }^{3}$ Although the production planning problem is complex in general, in this paper we disregard computational issues, and assume that an optimal plan can be found for every possible forecast.
} 
Figure 2 illustrates this mechanism design model. We are seeking such a mechanism, wherewith the performance of the supply network as a whole is optimal. This can be guaranteed, if all the agents disclose their private information truthfully, and the mechanism uses an optimal planning choice function. Let us define these properties formally.

Definition 1. A mechanism $\mathcal{M}$ is (weakly) truthful, if truth telling is a dominant strategy for every agent, i.e., it maximizes their expected utility: $\forall i, \forall \theta_{i} \in$ $\Theta, \forall \hat{\theta} \in \Theta^{n}, \forall \hat{c} \in C$ :

$$
\mathbb{E}_{\tilde{\theta}}\left[u_{i}\left(\theta_{i}, \tilde{\theta}, \hat{c}, \xi\right)\right] \geq \mathbb{E}_{\tilde{\theta}}\left[u_{i}\left(\theta_{i}, \hat{\theta}, \hat{c}, \xi\right)\right],
$$

where $\tilde{\theta}=\left(\hat{\theta}_{1}, \ldots, \hat{\theta}_{i-1}, \theta_{i}, \hat{\theta}_{i+1}, \ldots, \hat{\theta}_{n}\right)$, and $\forall c, \hat{c} \in C, \forall \hat{\theta} \in \Theta^{n}$ :

$$
\mathbb{E}_{\hat{\theta}}\left[u_{s}(c, \hat{\theta}, c, \xi)\right] \geq \mathbb{E}_{\hat{\theta}}\left[u_{s}(c, \hat{\theta}, \hat{c}, \xi)\right] .
$$

Definition 2. A mechanism $\mathcal{M}$ is efficient, if the choice function $f$ maximises the expected social welfare which is the sum of the utilities without the payments. Hence $\forall \theta \in \Theta^{n}, \forall c \in C$ :

$$
f(\theta, c) \in \underset{x \in \mathcal{K}}{\operatorname{argmax}} \mathbb{E}_{\theta}\left[\sum_{i=1}^{n} v_{i}(x, \xi)-c(x, \xi)\right] .
$$

Now we define the extensively used VCG mechanism for our supply network model, which is not only efficient, but in addition, it is truthful, since the agents are inspired to share their private information in order to align the choice function $f$ to their utility.

Definition 3. The mechanism $\mathcal{M}=\left(f, t_{1}, \ldots, t_{n}, t_{s}\right)$ is a VCG mechanism, if

$$
\begin{gathered}
f(\hat{\theta}, \hat{c}) \in \underset{x \in \mathcal{K}}{\operatorname{argmax}} \mathbb{E}_{\hat{\theta}}\left[\sum_{i=1}^{n} v_{i}(x, \xi)-\hat{c}(x, \xi)\right], \\
t_{i}(\hat{\theta}, \hat{c}, \xi)=h_{i}\left(\hat{\theta} \hat{\theta}_{-i}, \hat{c}, \xi\right)+\hat{c}(f(\hat{\theta}, \hat{c}), \xi)-\sum_{j \neq i} v_{j}(f(\hat{\theta}, \hat{c}), \xi), \text { and } \\
t_{s}(\hat{\theta}, \hat{c}, \xi)=\sum_{i=1}^{n} v_{i}(f(\hat{\theta}, \hat{c}), \xi)-h_{s}(\hat{\theta}, \xi),
\end{gathered}
$$

where $\hat{\theta}_{-i}=\left(\hat{\theta}_{1}, \ldots, \hat{\theta}_{i-1}, \hat{\theta}_{i+1}, \ldots, \hat{\theta}_{n}\right)$, furthermore $h_{s}$ and $h_{i}$ are arbitrary functions independent from the given agent's disclosed information.

The next theorem shows that if we are looking for an efficient and truthful mechanism, this excludes the possibility of cost sharing among the agents.

Theorem 1. Let $\mathcal{M}$ be an efficient and truthful mechanism. If $c, \hat{c} \in C$ and $\theta \in \Theta^{n}$ such that $f(\theta, c)=f(\theta, \hat{c})$ then $\forall \xi \in D: t_{s}(\theta, c, \xi)=t_{s}(\theta, \hat{c}, \xi)$. 
Proof. Due to Green and Laffont (1977), in an efficient and truthful mechanism the supplier's payment has to be in the form of Eq. (8). Since this does not depend directly on $c$, only on $f(\theta, c)$, this proves the theorem.

The theorem states that if two different cost functions result in the same production plan, then they also result in the same payment - even if one of them incurs higher cost for the supplier. The premise of the theorem is not artificial, for instance the newsvendor model (see Section 4) can involve different fixed costs for ordering, but the optimal order quantity depends only on the variable cost, therefore the same optimal lot size may incur different costs.

The case of the retailers is different, since their forecasts can be evaluated when the demands realise, thus their payments do not have to be in VCG form.

Theorem 2. If $f$ is efficient, $t_{s}$ is defined by Eq. (8) and the retailers' payments are

$$
t_{i}(\hat{\theta}, \hat{c}, \xi)=S_{i}\left(\hat{\theta}_{i}, \xi_{i}\right)+v_{i}(f(\hat{\theta}, \hat{c}), \xi)-h_{i}\left(\hat{\theta}_{-i}, \hat{c}, \xi\right),
$$

where $S_{i}$ is a strictly proper scoring rule and $h_{i}$ is an arbitrary function, then the mechanism is truthful.

Proof. Since $t_{s}$ is in the VCG form, it is a dominant strategy for the supplier to share the cost information truthfully. On the other hand, the expected utility of the retailers become

$$
\mathbb{E}_{\theta_{i}}\left[u_{i}\left(\theta_{i}, \hat{\theta}, \hat{c}, \xi\right)\right]=h_{i}\left(\hat{\theta}_{-i}, \hat{c}, \xi\right)-\mathbb{E}_{\theta_{i}}\left[S_{i}\left(\hat{\theta}_{i}, \xi_{i}\right)\right],
$$

which can be maximised by $\hat{\theta}_{i}=\theta_{i}$.

\subsection{Distributed implementation of the mechanism}

In the previous section we have presented a method for defining efficient and truthful mechanisms for the supply network. Now, our main goal is to omit the need for an independent mediator which does not comply with our basic requirements (see Section 3.1). However, at the same time, we are going to preserve the favourable properties of the system. In what follows, we dissolve the two reasons that called for the existence of an independent mechanism: (i) balancing the difference between the agents' payments, and (ii) providing efficiency.

Definition 4. A mechanism $\mathcal{M}$ is budget balanced, if $\forall \hat{\theta} \in \Theta^{n}, \forall \hat{c} \in C, \forall \xi \in$ $D^{n}$ :

$$
t_{s}(\hat{\theta}, \hat{c}, \xi)=\sum_{i=1}^{n} t_{i}(\hat{\theta}, \hat{c}, \xi)
$$

i.e., there is no surplus or deficit for the mechanism, or, with other words, the total payment is distributed among the agents. 
For the payments defined by Eq. (9) this means that

$$
t_{s}(\hat{\theta}, \hat{c}, \xi)=\sum_{i=1}^{n} v_{i}(f(\hat{\theta}, \hat{c}), \xi)-\sum_{i=1}^{n}\left[h_{i}\left(\hat{\theta}_{-i}, \hat{c}, \xi\right)-S_{i}\left(\hat{\theta}_{i}, \xi_{i}\right)\right] .
$$

Note that if the functions $h_{i}\left(\hat{\theta}_{-i}, \hat{c}, \xi\right)$ are independent of $\hat{c}$, then the resulted payment is in the VCG form, therefore we have an efficient, truthful and budget balanced mechanism. This is possible, since the private information of the retailers (the forecasts) can be verified after the demands realise. In contrast, in general mechanism design problems the three generic properties of truthfulness, efficiency and budget balance are exclusive (see e.g., Narahari et al., 2009).

This is similar to the result of Nisan and Ronen (2001), who introduced the concept of mechanisms with verification and proved that it is more powerful than classic mechanisms. Their task scheduling mechanism however does not involve stochastic variables and it does not aim at achieving efficiency.

Also note that the constructed mechanism can be implemented without an independent decision maker: the retailers share their forecasts with the supplier - just as it would be the mechanism-, and the utility-maximising behaviour of the supplier results in the overall efficient outcome. As long as the production plan is observable for the retailers, the cost can remain private information of the supplier, since the payments

$$
t_{i}(\hat{\theta}, \hat{c}, \xi)=S_{i}\left(\hat{\theta}_{i}, \xi_{i}\right)+v_{i}(f(\hat{\theta}, \hat{c}), \xi)-h_{i}\left(\hat{\theta}_{-i}, \xi\right)
$$

do not depend directly on $\hat{c}$, only on the plan $f(\hat{\theta}, \hat{c})$. The corresponding distributed setting is illustrated on Fig. 3.

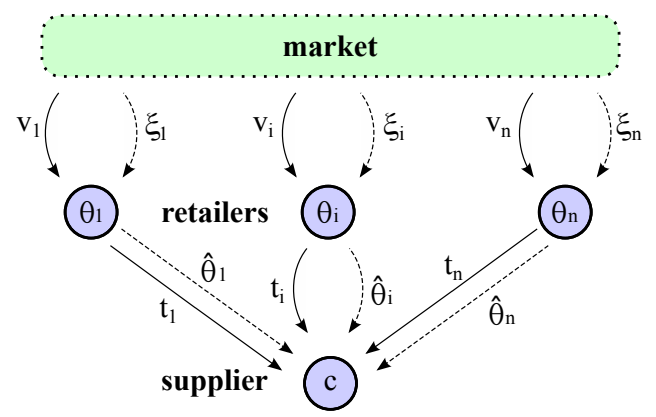

Figure 3: Supply network in a distributed mechanism design setting.

\subsection{Coordinating the network with distributed mechanisms}

In this section we specialise the previously developed mechanism for the VMI supply networks. Let us define the best possible income for retailer $i$ when the vector of the realised demands is $\xi$ :

$$
v_{i}^{*}(\xi)=\max _{x \in \mathcal{K}} v_{i}(x, \xi) .
$$


We consider $h_{i}\left(\hat{\theta}_{-i}, \xi\right)=v_{i}^{*}(\xi)-\beta_{i}\left(\xi_{i}\right)$, where $\beta_{i}$ is an arbitrary function. With this definition, the payment becomes

$$
t_{i}(\hat{\theta}, \hat{c}, \xi)=\beta_{i}\left(\xi_{i}\right)+S_{i}\left(\hat{\theta}_{i}, \xi_{i}\right)-\left(v_{i}^{*}(\xi)-v_{i}(x, \xi)\right) .
$$

This payment function has a rational interpretation that considers VMI a service. The first term is a payment for fulfilling the demand, the second term is a compensation for the forecast imprecision, while the last term is a negative compensation (penalty) for the lost sales that is paid by the supplier, if it cannot fulfil the demand. The suggested VMI supply protocol is shown by Fig. 4, while the next section illustrates the approach on a particular example.

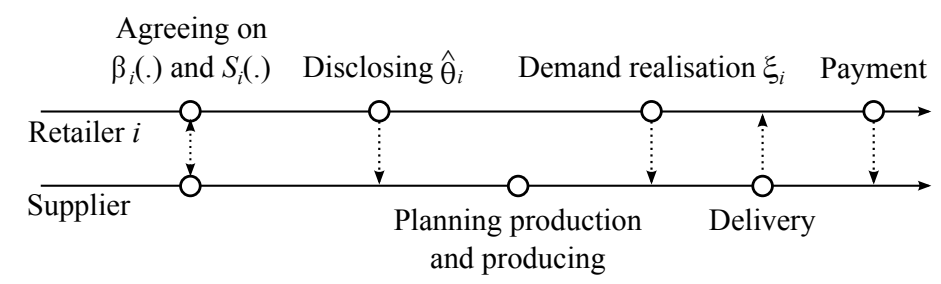

Figure 4: Timeline of the protocol.

\section{Example: the newsvendor model}

We consider the basic newsvendor model, where the single decision maker has to determine the production quantity $q$, while the demand $\xi$ is a random variable with known distribution ${ }^{4}$ (Qin et al., 2011). If $q>\xi$ then the excess inventory becomes obsolete, while $q<\xi$ causes lost sales. The profit in this model is $r \min (\xi, q)-c q$, where $r$ is the retail price and $c$ is the production cost. It is well known that the optimal production quantity maximizing the expected profit in this case is:

$$
q^{*}=\Phi^{-1}\left(\frac{r-c}{r}\right),
$$

where $\Phi$ is the cumulative density function of $\xi$, and for the sake of simplicity, we assume now that it is invertible.

Let us now return to the supply network model, where we illustrate on a particular example how the presented mechanism can improve the overall performance. We consider $n$ retailers, and assume that the $\xi_{i} \in \mathbb{R}$ demands are independent and normally distributed random variables with expected values $m_{i}$ and standard deviations $\sigma_{i}$. Let $\Phi_{i}$ denote the cumulative density function of $\xi_{i}$. In this case the production plan is $x=\left(q, q_{1}, \ldots, q_{n}\right)$, where $q \in \mathbb{R}$ is the production quantity, and $\left\{q_{i}: \mathbb{R}^{n} \rightarrow \mathbb{R}\right\}$ is the distribution plan. This latter defines the supply quantity $q_{i}(\xi)$ to retailer $i$ such that $\forall \xi: \sum_{i=1}^{n} q_{i}(\xi) \leq q$, i.e., the total quantity delivered cannot exceed the production quantity.

\footnotetext{
${ }^{4}$ In this special case, the demand vector $\xi$ has only one element.
} 


\subsection{Order-based purchase}

Firstly, we examine the traditional, suboptimal solution, where the retailers make firm orders $q_{i}$ and pay a $w$ wholesale price for the supplied goods, thus the payment becomes $t_{i}\left(q_{i}\right)=w q_{i}$. The supplier works in make-to-order mode, i.e., it produces the quantity $q=\sum q_{i}$ with $c$ piecewise cost. Since the retailers are in a newsvendor situation, their optimal order quantity can be determined by Eq. (16), but using $w$ instead of $c$, and $\Phi_{i}$ instead of $\Phi$. Since usually $w>c$, the order quantity is lower than the optimal (coordinated) one.

\subsection{Coordinated VMI}

In the VMI setting, the retailers should disclose their demand forecasts $\hat{\theta}_{i}=$ $\left(\hat{m}_{i}, \hat{\sigma}_{i}\right)$, and the supplier is responsible for determining the production quantity $q$, and distribution plan $\left\{q_{i}\right\}$. In this case the income of retailer $i$ becomes $v_{i}\left(q_{i}, \xi\right)=r \min \left(\xi_{i}, q_{i}\right)$, and $v_{i}^{*}(\xi)=r \xi_{i}$.

Let us define a payment in the form of Eq. (15). We use the following strictly proper scoring rule from Egri and Váncza (2011):

$$
S_{i}\left(\hat{m}_{i}, \hat{\sigma}_{i}, \xi_{i}\right)=\alpha_{i}\left(\frac{\left(\hat{m}_{i}-\xi_{i}\right)^{2}}{\hat{\sigma}_{i}}+\hat{\sigma}_{i}\right),
$$

where $\alpha_{i}>0$ is a constant, and furthermore we use $\beta_{i}\left(\xi_{i}\right)=w \xi_{i}$. Thus the payment becomes

$$
t_{i}\left(\hat{m}_{i}, \hat{\sigma}_{i}, \xi\right)=\left\{\begin{array}{ll}
w \xi_{i}+S_{i}\left(\hat{m}_{i}, \hat{\sigma}_{i}, \xi_{i}\right) & , \text { if } \xi_{i} \leq q_{i} \\
w q_{i}+S_{i}\left(\hat{m}_{i}, \hat{\sigma}_{i}, \xi_{i}\right)-(r-w)\left(\xi_{i}-q_{i}\right) & , \text { if } \xi_{i}>q_{i}
\end{array} .\right.
$$

This can be interpreted in the following way: the retailers should pay (i) for the supplied quantity, (ii) for the imprecision of the forecast, and (iii) if the supplied quantity does not cover the whole demand, the supplier must pay a shortage penalty. These three terms constitute the particular payment elements for the VMI service (as expressed in a general form by Eq. (15)).

With this payment function the retailers are inspired to truthfully reveal their private information about the expected values and standard deviations of the demand forecasts. Now, the expected value of the total demand will be the sum of the expected values, and since the demands are considered to be independent, the standard deviation of the total demand becomes $\sqrt{\sum \sigma_{i}^{2}}$. Furthermore, if the demand at the retailers are normally distributed, the distribution of the total demand will also be normal. If we let $\Phi$ denote the cumulative density function of the total demand, the optimal production quantity can be determined by Eq. (16). As for the distribution plan, if the total realised demand is less than the produced quantity, the total demand should be fulfilled, otherwise, the total produced quantity should be distributed among the retailers in an arbitrary way. 


\subsection{Participation}

In this section we study the participation requirement, thus we compare the expected utilities of the partners in the order based and in the VMI cases. Further on, we will assume that the $\alpha_{i}$ parameters are equal, thus we will omit its index. This assumption means that the supplier does not discriminate the retailers, each retailer has the same wholesale price and scoring rule. The retailers' utility considering ordering and VMI are

$$
u_{i}^{\mathrm{ORD}}=r \min \left(\xi_{i}, q_{i}^{*}\right)-w q_{i}^{*},
$$

and

$$
u_{i}^{\mathrm{VMI}}=(r-w) \xi_{i}-\alpha\left(\frac{\left(m_{i}-\xi_{i}\right)^{2}}{\sigma_{i}}+\sigma_{i}\right),
$$

where $q_{i}^{*}=\Phi_{i}^{-1}\left(\frac{r-w}{r}\right)$. The expected utilities therefore become

$$
\begin{aligned}
\mathbb{E}\left[u_{i}^{\mathrm{ORD}}\right]=r \mathbb{E}\left[\min \left(\xi_{i}, q_{i}^{*}\right)\right]-w q_{i}^{*}=r \int_{-\infty}^{q_{i}^{*}} x \Phi_{i}^{\prime}(x) \mathbf{d} x+r q_{i}^{*} \int_{q_{i}^{*}}^{\infty} \Phi_{i}^{\prime}(x) \mathbf{d} x \\
-w q_{i}^{*}=r \int_{-\infty}^{q_{i}^{*}} x \Phi_{i}^{\prime}(x) \mathbf{d} x+r q_{i}^{*}\left(1-\Phi_{i}\left(q_{i}^{*}\right)\right)-w q_{i}^{*} \\
=r \int_{-\infty}^{q_{i}^{*}} x \Phi_{i}^{\prime}(x) \mathbf{d} x+r q_{i}^{*}\left(\frac{w}{r}\right)-w q_{i}^{*}=r \int_{-\infty}^{q_{i}^{*}} x \Phi_{i}^{\prime}(x) \mathbf{d} x,
\end{aligned}
$$

and

$$
\mathbb{E}\left[u_{i}^{\mathrm{VMI}}\right]=\mathbb{E}\left[(r-w) \xi_{i}-\alpha\left(\frac{\left(m_{i}-\xi_{i}\right)^{2}}{\sigma_{i}}+\sigma_{i}\right)\right]=(r-w) m_{i}-2 \alpha \sigma_{i},
$$

since $\mathbb{E}\left[\left(m_{i}-\xi_{i}\right)^{2}\right]=\sigma_{i}^{2}$. Thus the retailer prefers VMI if

$$
\alpha \leq \frac{(r-w) m_{i}-r \int_{-\infty}^{q_{i}^{*}} x \Phi_{i}^{\prime}(x) \mathbf{d} x}{2 \sigma_{i}} .
$$

Definition 5. Let us define the Gauss error function as

$$
\operatorname{erf}(x)=\frac{2}{\sqrt{\pi}} \int_{0}^{x} e^{-t^{2}} \mathbf{d} t
$$

and let $\operatorname{erf}^{-1}(x)$ denote its inverse.

Theorem 3. The retailers prefer VMI if

$$
\alpha \leq \frac{r e^{-\left(e r f^{-1}\left(2 \frac{r-w}{r}-1\right)\right)^{2}}}{2 \sqrt{2 \pi}} .
$$

Proof. The inverse of the cumulative density function of the normal distribution can be written in the form

$$
\Phi_{i}^{-1}(x)=m_{i}+\sqrt{2} \sigma_{i} \operatorname{erf}^{-1}(2 x-1),
$$


thus applying the Newton-Leibniz formula we get

$$
\int_{-\infty}^{q_{i}^{*}} x \Phi_{i}^{\prime}(x) \mathbf{d} x=\int_{-\infty}^{\Phi_{i}^{-1}\left(\frac{r-w}{r}\right)} x \Phi_{i}^{\prime}(x) \mathbf{d} x=-\frac{e^{-\left(\operatorname{erf}^{-1}\left(2 \frac{r-w}{r}-1\right)\right)^{2}}}{\sqrt{2 \pi}} \sigma_{i}+m_{i} \frac{r-w}{r} .
$$

Substituting this result into Eq. (23) we prove the statement of the theorem.

Since the condition of Theorem 3 depends only on commonly known parameters, it can be evaluated by every agents and it can be considered during the contract negotiation phase.

Let us examine the participation of the supplier now. Its (expected) utility when ordering is

$$
\mathbb{E}\left[u_{s}^{\mathrm{ORD}}\right]=(w-c) \sum_{i=1}^{n} q_{i}^{*},
$$

while in the VMI case it is

$$
\mathbb{E}\left[u_{s}^{\mathrm{VMI}}\right]=2 \sum_{i=1}^{n} \alpha \sigma_{i}-(r-w) \sum_{i=1}^{n} m_{i}+r \int_{-\infty}^{q^{*}} x \Phi^{\prime}(x) \mathbf{d} x,
$$

where $q^{*}=\Phi^{-1}\left(\frac{r-c}{r}\right)$.

Theorem 4. The supplier prefers VMI if

$$
\alpha \geq(w-c) \frac{\sqrt{2} \operatorname{erf}^{-1}\left(2 \frac{r-w}{r}-1\right)}{2}+\frac{r e^{-\left(e r f^{-1}\left(2 \frac{r-c}{r}-1\right)\right)^{2}}}{2 \sqrt{2 \pi}} \frac{\sqrt{\sum_{i=1}^{n} \sigma_{i}^{2}}}{\sum_{i=1}^{n} \sigma_{i}} .
$$

Proof. Using Eq. (26) we get

$$
\mathbb{E}\left[u_{s}^{\mathrm{ORD}}\right]=(w-c)\left(\sum_{i=1}^{n} m_{i}+\sqrt{2} \operatorname{erf}^{-1}(2 x-1) \sum_{i=1}^{n} \sigma_{i}\right)
$$

and

$\mathbb{E}\left[u_{s}^{\mathrm{VMI}}\right]=2 \alpha \sum_{i=1}^{n} \sigma_{i}-(r-w) \sum_{i=1}^{n} m_{i}-\frac{r e^{-\left(\operatorname{erf}^{-1}\left(2 \frac{r-c}{r}-1\right)\right)^{2}}}{\sqrt{2 \pi}} \sqrt{\sum_{i=1}^{n} \sigma_{i}^{2}+(r-c) \sum_{i=1}^{n} m_{i} .}$

From this two results we get the statement of the theorem.

Note that the condition of Theorem 4 can be evaluated neither by the retailers (since they do not know $c$ ), nor by the supplier when $n>1$ (since the $\sigma_{i}$ values are known only by the retailers). However, exploiting that $\sqrt{\sum_{i=1}^{n} \sigma_{i}^{2}} \leq$ $\sum_{i=1}^{n} \sigma_{i}$, we can relax the bound and the following condition can then be evaluated by the supplier: 
Corollary 1 (Sufficient condition). The supplier prefers VMI if

$$
\alpha \geq(w-c) \frac{\sqrt{2} e r f^{-1}\left(2 \frac{r-w}{r}-1\right)}{2}+\frac{r e^{-\left(e r f^{-1}\left(2 \frac{r-c}{r}-1\right)\right)^{2}}}{2 \sqrt{2 \pi}} .
$$

The retailers and the supplier can use Theorem 3 and Corollary 1 respectively for guaranteeing expected benefit in the VMI compared to the order based purchase during the negotiation about parameters $w$ and $\alpha$. Note that this result is limited to this special model only and we cannot provide such guarantees in the general case.

\subsection{Simulation study}

We compared the performance of the order-based and coordinated VMI models in a series of computational experiments. Fig. 5 illustrates the difference between the total network utility $\left(U^{\mathrm{VMI}}=u_{s}^{\mathrm{VMI}}+\sum u_{i}^{\mathrm{VMI}}\right.$ and $U^{\mathrm{ORD}}=$ $\left.u_{s}^{\mathrm{ORD}}+\sum u_{i}^{\mathrm{ORD}}\right)$ of the two approaches presented in sections 4.1 and 4.2 , depending on the number of retailers. We set the price, payment and cost parameters as $c=50, w=70$, and $r=100$ (note that $U^{\mathrm{VMI}}$ is independent of $\alpha_{i}$, therefore they are not specified here). The expected value and standard deviation of the total demand was set to $m=800$ and $\sigma=100$, and we considered retailers with identical distributions, therefore their parameters were $m_{i}=m / n$ and $\sigma_{i}=\sigma / \sqrt{n}$. Each cost value indicated on the figures in this and the next subsections are averages made on 5000 simulation runs.

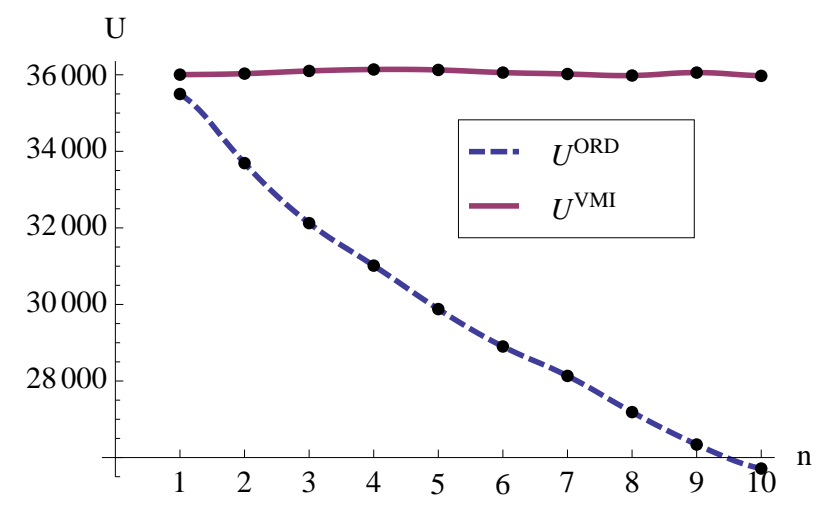

Figure 5: The network utility in function of the number of retailers.

As it can be seen on Fig. 5, the coordinated VMI is more efficient than the order-based supply even in the single retailer case due to the elimination of the double marginalisation. However, when the number of the retailers increases, risk pooling keeps the optimality of the network, while the uncoordinated approach quickly deviates from cost efficient performance. 


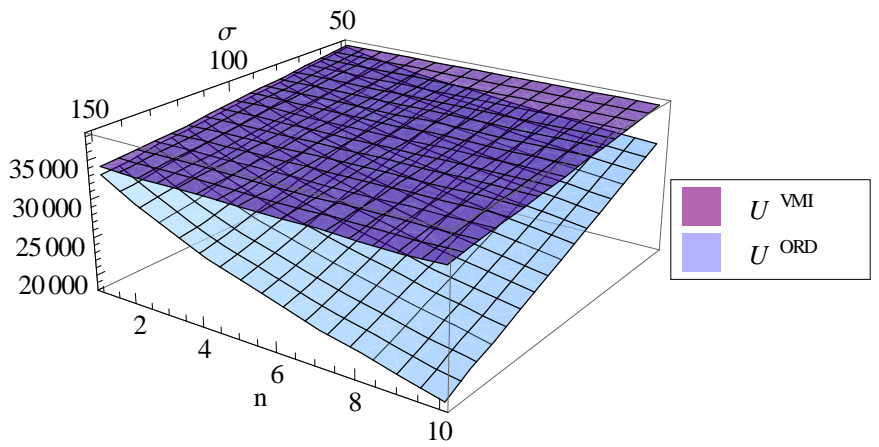

Figure 6: The network utility in function of the number of retailers and the standard deviation.

Fig. 6 gives further insight into the behaviour of the network when not only the number of retailers, but also the uncertainty of the forecast increase. The upper surface on the figure depicts the total utility in the coordinated VMI case. As we have shown in Fig. 5, the performance in this case is independent of the number of retailers. However, increasing uncertainty (standard deviation) of forecasts decreases the utility, as it is expected. The lower surface, on the other hand, illustrates the total utility of order-based supply. Here, compared to the coordinated solution, network performance is drastically deteriorated either with the growing number of retailers or with the increase of the uncertainty of forecasts. In this case, risk pooling is not at work.

\subsection{Sensitivity analysis}

Fig. 7 shows the result of changing the $\alpha_{1}$ parameter of the scoring rule in Eq. (17) in the single retailer case. Since $\alpha_{1}$ does not influence the order-based purchase, the utility is constant then. It can be seen, that when $\alpha_{1}$ is around 16 , both VMI utilities are greater compared to the ordering approach, therefore both agents prefer VMI. If $\alpha_{1}$ is approximately 30, the utility of the retailer and the supplier in VMI equals - however, the retailer obtains lower utility than in the ordering case.

Fig. 8 illustrates the participation constraints of the network members with the same $c, w$ and $r$ parameters as before. It can be observed that in the single retailer case we get the lower and upper bounds that can be seen on Fig. 7. Furthermore, since the standard deviation of the retailer's and the total demand are the same, the estimation of the lower bound is strict. Increasing the number of the retailers does not influence the upper bound, but decreases the lower bound in accordance with Theorems 3 and 4 . The estimated lower bound is naturally equal to the lower bound in the single retailer case. 


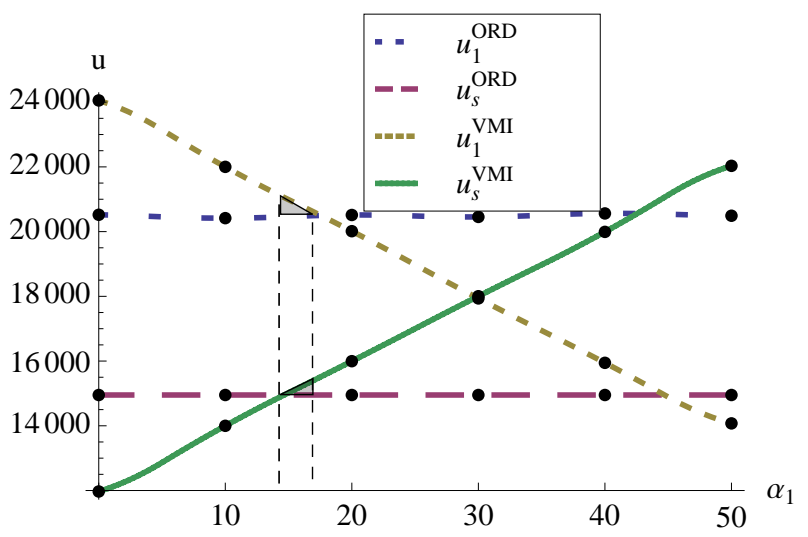

Figure 7: The agents' utilities in function of $\alpha_{1}$.

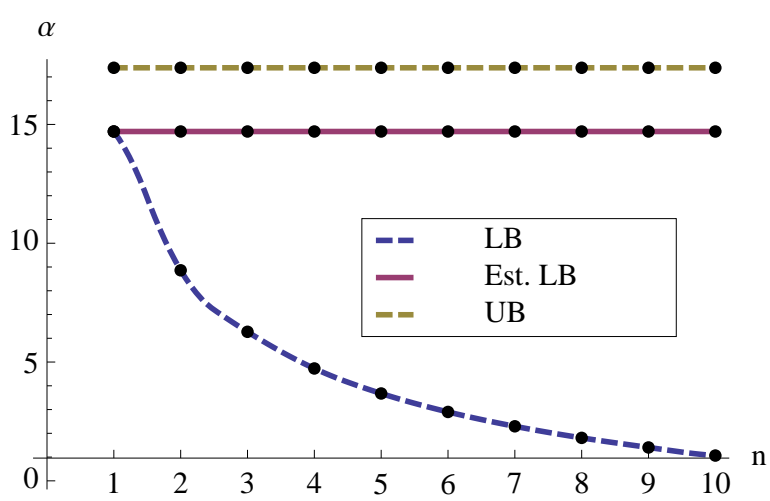

Figure 8: Upper, lower and estimated lower bounds on $\alpha$.

\section{Discussion and conclusion}

We have presented a general mechanism design model for coordinating a supply network of a single supplier and multiple retailers, and proved a negative result for cost sharing. The mechanism that was discussed first in an abstract form has also been specialised by taking practical characteristics of supply networks into consideration. In light of the results we suggested to interpret VMI as a flexible service. It was shown that the coordination protocol needs no independent decision maker for guaranteeing truthfulness and efficiency of the network. We also have proved that the actual cost structure of the supplier can remain private information, i.e., the mechanism respects the asymmetry of sensitive cost information.

As discussed above, these generic properties are essential in any real appli- 
cation. However, before transferring the results into practice we suggest considering the following issues:

- The production plan in the model is very general, and can contain several aspects of the practical problems, such as production quantities, production time, production technologies, and distribution plan, to name a few. Even the retail price can be part of the plan, thus the model allows price-dependent stochastic demand as well. Some examples of the different planning problems are the classic newsvendor (Section 4), the newsvendor with costly additional production possibility (Egri and Váncza, 2011), the newsvendor with additional production involving fixed setup cost (Egri and Váncza, 2012) and the multi-period uncapacitated lot sizing (Váncza et al., 2008).

- While in the general model the whole production plan and the demand vector is assumed to be common knowledge, this is usually not necessary in the specific applications. By exploiting the structure of $v_{i}$ and $x$, this assumption can be often relaxed. In the example presented in Section 4, where $v_{i}$ depends only on demand $\xi_{i}$ and the supplied quantity $q_{i}$, the payment is constructed in such a way that $\xi_{i}$ and $q_{i}$ have to be known only by retailer $i$ and the supplier. This property facilitates the practical applicability of the model.

- Although some kind of cost and profit sharing would be favourable, unfortunately in general networks with private information this cannot be guaranteed. Furthermore, even positive utilities for the agents are not assured. However, usually the combination of the widely used wholesale price and a relatively small compensation for the forecast imprecision can coordinate the network. Simulations and scenario analyses based on historical data and beliefs can help evaluate and tune the parameters.

In our opinion the conceptual apparatus and research methodology of mechanism design can have a fundamental role in coordinating networked production. Within the context of the work presented in this paper, we see some research directions particularly worth exploring. Hence, the presented two-tier network can be extended to more complex structures, where the centralised decision making is less realistic. One can also explicitly consider more complex planning problems that cannot be solved optimally, and the approximation distorts the favourable properties of the mechanisms (see Nisan and Ronen, 2001). In such cases interactive planning protocols seem to be applicable. As for distributed decision making, in the context of supply chain design we have recently taken the mechanism design approach to suggesting a solution of the safety stock placement problem in decentralised supply chains consisting of a number of autonomous stages (Egri, 2012).

Finally we note that the practical implementation of the proposed VMI supply service calls for new legal instruments, contract types that come along with tedious processes of elaboration and introduction. What is more, it also needs 
a shift in the general managerial attitude towards taking more responsibility for the information communicated to business partners. Against all such difficulties, one can but hope that the potential mutual merits of coordination makes the approach attractive on the long run.

\section{Acknowledgements}

This work has been supported by the OMFB No. 01638/2009 and the TÁMOP4.2.2.B-10/1-2010-0009 grants. Péter Egri acknowledges the support of the János Bolyai scholarship No. BO/00659/11/6. The authors thank the three anonymous reviewers for their helpful suggestions.

\section{References}

Cachon, G.P. (2003). Supply chain coordination with contracts. In: de Kok, A.G., Graves, S.C., (Eds.). Supply Chain Management: Design, Coordination and Cooperation. Handbooks in Operations Research and Management Science, 11. Elsevier, 229-339.

Chen, J. \& Bell, P.C. (2011). Coordinating a decentralized supply chain with customer returns and price-dependent stochastic demand using a buyback policy. European Journal of Operational Research, 212(2), 293-300.

Chen, K. \& Xiao, T. (2011). Ordering policy and coordination of a supply chain with two-period demand uncertainty. European Journal of Operational Research, 215(2), 347-357.

Drótos, M., Erdős, G. \& Kis, T. (2009). Computing lower and upper bounds for a large-scale industrial job shop scheduling problem. European Journal of Operational Research, 197(1), 296-306.

Egri, P. (2012). Safety stock placement in non-cooperative supply chains. Proc. of the 3rd Workshop on Artificial Intelligence and Logistics (AILog2012), 20th European Conference on Artificial Intelligence (ECAI), 31-36.

Egri, P. \& Váncza, J. (2011). Supply network coordination by Vendor Managed Inventory - A mechanism design approach. Proc. of the 2nd Workshop on Artificial Intelligence and Logistics (AILog-2011), 22nd International Joint Conference on Artificial Intelligence (IJCAI), 19-24.

Egri, P. \& Váncza, J. (2012). Channel coordination with the newsvendor model using asymmetric information. International Journal of Production Economics, 135(1), 491-499.

Esmaeili, M., Aryanezhad, M.-B. \& Zeephongsekul, P. (2009). A game theory approach in seller-buyer supply chain. European Journal of Operational Research, 195(2), 442-448. 
Feldmann, M. \& Müller, S. (2003). An incentive scheme for true information providing in Supply Chains. Omega, 31(2), 63-73.

Gan, X., Sethi, S.P. \& Zhou, J. (2010). Commitment-penalty contracts in dropshipping supply chains with asymmetric demand information. European Journal of Operational Research, 204(3), 449-462.

Garg, D., Narahari, Y., Foster, E., Kulkarni, D.M. \& Tew, J.D. (2005). A Groves mechanism approach to decentralized design of supply chains. Proc. of the IEEE Conference on Electronic Commerce, 330-337.

Green, J. \& Laffont, J-J. (1977). Characterization of satisfactory mechanisms for the revelation of preferences for public goods. Econometrica, 45(2), 427-438.

Li, X., Wang, Q. (2007). Coordination mechanisms of supply chain systems. European Journal of Operational Research, 179(1), 1-16.

Liu, H. \& Özer, Ö. (2010). Channel incentives in sharing new product demand information and robust contracts. European Journal of Operational Research, 207(3), 1341-1349.

Nagarajan, M. \& Sošić, G. (2008). Game-theoretic analysis of cooperation among supply chain agents: review and extensions. European Journal of $O p$ erational Research, 187(3), 719-745.

Narahari, Y., Garg, D., Narayanam, R. \& Prakash, H. (2009). Game theoretic problems in network economics and mechanism design solutions. London: Springer, (Chapter 2).

Nisan, N. \& Ronen, A., (2001). Algorithmic mechanism design. Games and Economic Behavior, 35, 166-196.

Qin, Y., Wang, R., Vakharia, A.J., Chen, Y. \& Seref, M.M.H. (2011). The newsvendor problem: Review and directions for future research. Invited review. European Journal of Operational Research, 213(2), 361-374.

Savage, L.J. (1971). Elicitation of personal probabilities and expectations. Journal of the American Statistical Association, 66(336), 783-801.

Simchi-Levi, D., Kaminsky, P. \& Simchi-Levi, E. (2000). Designing and managing the supply chain: Concepts, strategies, and cases. New York: McGrawHill.

Váncza, J., Egri, P. \& Monostori, L. (2008). A coordination mechanism for rolling horizon planning in supply networks. CIRP Annals - Manufacturing Technology, 57(1), 455-458.

Váncza, J., Monostori, L., Lutters, D., Kumara, S.R., Tseng, M., Valckenaers, P. \& Van Brussel, H. (2011). Cooperative and responsive manufacturing enterprises. CIRP Annals - Manufacturing Technology, 60(2), 797-820. 
Wang, H., Guo, M. \& Efstathiou, J. (2004). A game-theoretical cooperative mechanism design for a two-echelon decentralized supply chain. European Journal of Operational Research, 157(2), 372-388.

Wang, J.-C., Lau, H.-S. \& Lau, A.H.L. (2009). When should a manufacturer share truthful manufacturing cost information with a dominant retailer? European Journal of Operational Research, 197(1), 266-286.

Yu, Y., Chu, F. \& Chen, H. (2009). A Stackelberg game and its improvement in a VMI system with a manufacturing vendor. European Journal of Operational Research, 192(3), 929-948.

Yu, Y. \& Huang, G.Q. (2010). Nash game model for optimizing market strategies, configuration of platform products in a Vendor Managed Inventory (VMI) supply chain for a product family. European Journal of Operational Research, 206(2), 361-373.

Zohar, A. \& Rosenschein, J.S. (2008). Mechanisms for information elicitation. Artificial Intelligence, 172(16-17), 1917-1939. 